\title{
ВЫЧИСЛЕНИЕ ЭХО-СИГНАЛА ОТ ЦИЛИНДРИЧЕСКОЙ ОБОЛОЧКИ ПРОИЗВОЛЬНОГО ПОПЕРЕЧНОГО СЕЧЕНИЯ
}

Дифракция акустических и электромагнитных волн на цилиндрических телах эллиптической либо параболической формы исследовалась в ряде работ. При этом эти тела рассматривались как «акустически жесткие» или «мягкие» $\left[{ }^{1-4}\right]$. Дифракция волн на «акустически жестких» и «мягких» объектах более сложной формы изучалась в $\left[{ }^{5-8}\right]$. Решение задачи отыскивалось в $\left[{ }^{5}\right]$ методом парциальных волн, в $\left[{ }^{7}\right]-$ вариационными методами, в $\left.{ }^{8}\right]$ - методом наименьших квадратов. В $\left[{ }^{9}\right]$ давление, рассеянное от упругого тела, вычислялось с помощью интегральной формулы Кирхгофа.

В настоящей работе для вычисления эхо-сигнала, вызванного плоским зондирующим импульсом, от тонкой цилиндрической оболочки произвольного сечения, находящейся в безграничной жидкой среде, строится алгоритм, в основу которого положен метод Бубнова-Галеркина. В пространстве преобразования Фурье решение отыскивается в полярной системе координат $r, \vartheta, \tau$ в виде конечных рядов по расходящимся волнам. Длина безразмерного радиус-вектора $r$ измеряется в толщинах $h$ оболочки, безразмерное время $\tau$ вычисляется по формуле $\tau=c t / h$, где $t-$ время и $c-$ скорость звука в среде. Начало координат выбрано так, что поверхность цилиндрической оболочки определяется однозначно уравнением

$$
r=R(\vartheta) .
$$

При $\vartheta=0$ радиус-вектор направлен перпендикулярно к фронту зондирующего импульса в направлении, обратном к распространению его фронта. Отсчет времени выбран так, что при $\tau=0$ фронт зондирующего импульса находится на безразмерном расстоянии $r_{0}$ от начала координат.

Для описания распространения зондирующего импульса $p_{i}$ и эхосигнала $p_{e}$ в среде, окружающей оболочку, используется уравнение движения идеальной сжимаемой жидкой среды в акустическом приближении. В таком приближении зондирующий импульс $p_{i}$ в координатах $l$, $\tau$ и эхо-сигнал $p_{e}$ в координатах $r, \vartheta, \tau$ удовлетворяют соответственно уравнениям

$$
\begin{aligned}
& \frac{\partial^{2} p_{i}}{\partial l^{2}}-\frac{\partial^{2} p_{i}}{\partial \tau^{2}}=0 \\
& \nabla^{2} p_{e}-\frac{\partial^{2} p_{e}}{\partial \tau^{2}}=0
\end{aligned}
$$

где $l$ - безразмерное расстояние, измеряемое от плоскости отсчета (от плоскости, где расположен фронт зондирующего импульса при $\tau=0)$, и 


$$
\nabla^{2}=\frac{\partial^{2}}{\partial r^{2}}+\frac{1}{r} \frac{\partial}{\partial r}+\frac{1}{r^{2}} \frac{\partial^{2}}{\partial \vartheta^{2}} .
$$

Зондирующий импульс, удовлетворяющий уравнению (2), выбран в виде

$$
p_{i}(l, \tau)=p_{*} g(\tau-l)\left[H(\tau-l)-H\left(\tau-l-\tau_{*}\right)\right],
$$

где $p_{*}$ - постоянная, определяющая амплитуду зондирующего импульca, $\tau$ * - безразмерная продолжительность зондирующего импульса, $g(\tau)$ - закон изменения давления в зондирующем импульсе, $H(\tau)$ единичная функция Хевисайда.

Для представления импульса (5) в координатах $r$, $\vartheta$ следует воспользоваться соотношением

$$
l=r_{0}-r \cos \vartheta
$$

Векторы перемещений среды $\mathbf{w}_{i}, \mathbf{w}_{e}$, вызванные давлениями $p_{i}, p_{e}$, определяются через эти давления соотношениями

$$
\frac{\partial^{2} \mathbf{w}_{i}}{\partial \tau^{2}}=-\frac{h}{\varrho c^{2}} \operatorname{grad} p_{i}, \quad \frac{\partial^{2} \mathbf{w}_{e}}{\partial \tau^{2}}=-\frac{h}{\varrho c^{2}} \operatorname{grad} p_{e},
$$

где $\varrho-$ плотность среды.

Для описания волн деформации в цилиндрической оболочке произвольного поперечного сечения используются уравнения теории типа Тимошенко $\left[{ }^{10}\right]$

$$
L_{i j} u_{j}=-\delta_{i 3} q \alpha^{-1} Q^{-1} c^{-2}, \quad i=1,2,3,
$$

где суммирование от 1 до 3 производится по индексу $j$.

В формуле (8) $\delta_{i j}-$ символ Кронекера, $q-$ нормальное давление, действующее на поверхность оболочки, и

$$
\begin{aligned}
L_{11} & =\frac{\partial^{2}}{\partial s^{2}}-x^{2} \mu^{2}-\beta^{-2} \frac{\partial^{2}}{\partial \tau^{2}}, \quad L_{12}=x^{2} \mu, \\
L_{13} & =\left(1+x^{2}\right) \mu \frac{\partial}{\partial s}+\frac{\partial \mu}{\partial s}, \quad L_{21}=x^{2} \mu, \\
L_{22} & =\frac{1}{12}\left(\frac{\partial^{2}}{\partial s^{2}}-\beta^{-2} \frac{\partial^{2}}{\partial \tau^{2}}\right)-x^{2}, \quad L_{23}=-x^{2} \frac{\partial}{\partial s}, \\
L_{31} & =-\left(1+x^{2}\right) \mu \frac{\partial}{\partial s}-\frac{\partial \mu}{\partial s}, \quad L_{32}=x^{2} \frac{\partial}{\partial s}, \\
L_{33} & =-\mu^{2}+x^{2} \frac{\partial^{2}}{\partial s^{2}}-\beta^{-2} \frac{\partial^{2}}{\partial \tau^{2}}, \\
u_{1} & =u / h, \quad u_{2}=\psi, \quad u_{3}=w / h,
\end{aligned}
$$

где $u, w, \psi$ - касательное и нормальное перемещения и угол поворота поперечного сечения оболочки. Кривизна срединной поверхности оболочки $\mu(\vartheta)$, входящая в операторы (9), вычисляется по формуле

$$
\mu(\vartheta)=B(\vartheta) A^{-1}(\vartheta),
$$

где

$$
\begin{aligned}
& A(\vartheta)=\left[R^{2}(\vartheta)+T^{2}(\vartheta)\right]^{1 / 2}, \quad T(\vartheta)=d R(\vartheta) / d \vartheta, \\
& B(\vartheta)=\left[R^{2}(\vartheta)+2 T^{2}(\vartheta)-R(\vartheta) d T(\vartheta) / d \vartheta\right] A^{-2}(\vartheta) .
\end{aligned}
$$


Элемент дуги $d s$ в формулах (9) определяется через $d \vartheta$ соотношением

$$
d s=A(\vartheta) d \vartheta
$$

Постоянные, входящие в уравнения (8) и операторы (9), имеют вид

$$
\alpha=\varrho_{1} \varrho^{-1} \beta^{2}, \quad \beta=c^{-1}\left[E /\left(1-v^{2}\right) \varrho_{1}\right]^{1 / 2}, \quad \chi=\left[k_{T}(1-v) / 2\right]^{1 / 2},
$$

где $\varrho_{1}, E, v$ - плотность, модуль упругости и коэффициент Пуассона материала оболочки, $k_{T}$ - коэффициент сдвига в теории типа Тимошенко $\left(k_{T}^{2}=0,86\right)$.

Зондирующий импульс (5), падая на цилиндрическую оболочку и рассеиваясь, порождает в ней упругие волны, которые, в свою очередь, возбуждают излученное давление в окружающей оболочку среде. Для вычисления эхо-сигнала с учетом деформативности оболочки следует отыскать решение типа расходящейся волны уравнения (3) при условии контакта

$$
\frac{\partial^{2}}{\partial \tau^{2}}\left[\left(\mathbf{w}_{i}+\mathbf{w}_{e}\right) \cdot \mathrm{n}\right]_{r=R}=\frac{\partial^{2} w}{\partial \tau^{2}}
$$

где $\mathbf{n}(\vartheta)$ - вектор нормали поверхности $r=R(\vartheta)$. Нормальное перемещение w оболочки в формуле (14) определяется с помощью уравнений (8), причем давление, действующее на поверхность оболочки, вычисляется при этом по формуле

$$
q=-\left[p_{i}+p_{e}\right]_{r=R} .
$$

Определив вектор нормали поверхности $r=R(\vartheta)$ через функцию $R(\vartheta)$ в виде

$$
\mathbf{n}(\vartheta)=\left[R(\vartheta) \mathbf{e}_{r}-T(\vartheta) \mathbf{e}_{\diamond}\right] A^{-1}(\vartheta),
$$

где $\mathrm{e}_{r}, \mathrm{e}_{f}$ - орты полярной системы координат, и воспользовавшись соотношениями (7), можем представить условие контакта (14) в виде

$$
\left[R \frac{\partial p_{e}}{\partial r}-\frac{T}{R} \frac{\partial p_{e}}{\partial \vartheta}\right]_{r=R}+\varrho c^{2} A \frac{\partial^{2} u_{3}}{\partial \tau^{2}}=F
$$

где $F(\tau, \vartheta)$ вычисляется по формуле

$$
F=-\left[R \frac{\partial p_{i}}{\partial r}-\frac{T}{R} \frac{\partial p_{i}}{\partial \vartheta}\right]_{r=R}
$$

Для решения уравнений (3) и (8) используется преобразование Фурье по времени в виде

$$
\begin{aligned}
& p(r, \vartheta, \tau)=\frac{1}{2 \pi} \int_{-\infty}^{\infty} p^{F}(r, \vartheta ; \omega) \mathrm{e}^{-i \omega \tau} d \omega, \\
& p^{F}(r, \vartheta ; \omega)=\int_{-\infty}^{\infty} p(r, \vartheta, \tau) \mathrm{e}^{i \omega \tau} d \tau .
\end{aligned}
$$

На основе (20) зондирующий импульс (5) определяется в пространстве преобразования Фурье в виде

$$
p_{i}^{F}(r, \vartheta ; \omega)=p_{*} g^{F}(\omega) \mathrm{e}^{i \omega\left(r_{0}-r \cos \vartheta\right)},
$$




$$
g^{F}(\omega)=\int_{0}^{\tau_{*}} g(\tau) \mathrm{e}^{i \omega \tau} d \tau
$$

Решение уравнений (3) и (8) в пространстве преобразования Фурье отыскивается методом Бубнова-Галеркина. Для этого изображение решения типа расходящейся волны уравнения (3) будем искать в конечных рядах по собственным функциям. С учетом формулы (21) оно определяется соотношением

$$
p_{e}^{F}(r, \vartheta ; \omega)=p_{*} g^{F}(\omega) \mathrm{e}^{i \omega r_{0}} \sum_{n=0}^{N} H_{n}(\omega r)\left[x_{n}^{1}(\omega) \sin n \vartheta+x_{n}^{2}(\omega) \cos n \vartheta\right] .
$$

Изображение решения системы (8) будем искать в виде рядов

$$
\begin{aligned}
& u_{1}^{F}(s ; \omega)=p \cdot Q^{-1} c^{-2} g^{F}(\omega) \mathrm{e}^{i \omega r_{0}} \sum_{n=0}^{N} x_{n}^{3}(\omega) \cos a n s+x_{n}^{4}(\omega) \sin \text { ans, } \\
& u_{2}^{F}(s ; \omega)=p \cdot Q^{-1} c^{-2} g^{F}(\omega) \mathrm{e}^{i \omega r_{0}} \sum_{n=0}^{N} x_{n}^{5}(\omega) \cos a n s+x_{n}^{6}(\omega) \sin a n s, \\
& u_{3}^{F}(s ; \omega)=p * Q^{-1} c^{-2} g^{F}(\omega) \mathrm{e}^{i \omega r_{0}} \sum_{n=0}^{N} x_{n}^{7}(\omega) \sin a n s+x_{n}^{8}(\omega) \cos \text { ans. }
\end{aligned}
$$

Здесь $x_{0}^{1}=x_{0}^{4}=x_{0}^{6}=x_{0}^{7}=0, \quad H_{n}(\omega r)$ - функция Ханкеля первого рода и

$$
a=2 \pi / L, \quad L=\int_{0}^{2 \pi} A(\vartheta) d \vartheta .
$$

Длина дуги $s$ определяется на основе формулы (12) с помощью интеграла

$$
s=\int_{0}^{\vartheta} A(\vartheta) d \vartheta
$$

Поскольку каждый член ряда (23) удовлетворяет уравнению (3) в пространстве преобразования Фурье, то для определения коэффициентов $x_{n}^{k}(\omega), k=1,2, \ldots, 8$, достаточно, чтобы выполнялись уравнения (8) и условия контакта (17).

Подставляя выражения (23) и (24) в систему (8), учитывая формулу (15) и условия контакта (17) в пространстве преобразования Фурье, умножая все уравнения полученной системы на $\sin a m s$ и $\cos a m s, m=0,1,2, \ldots, N$, и интегрируя их по $s$ от 0 до $L$, получим для определения коэффициентов $x_{n}^{k}(\omega), k=1,2, \ldots, 8, n=0,1,2, \ldots$ $\ldots, N$, алгебраическую систему, состоящую из $8 N+4$ уравнений. Зависимость $\vartheta=\vartheta(s)$ при интегрировании по $s$ определяется из соотношения (26).

Преобразование Фурье обращается численно по формуле

$$
\begin{gathered}
p_{e}(r, \vartheta, \tau)=\frac{p_{*}}{2 \pi} \int_{\omega_{A}}^{\omega_{B}} g^{F}(\omega) \mathrm{e}^{-i \omega\left(\tau-r_{0}\right)} \sum_{n=0}^{N} H_{n}(\omega r)\left[x_{n}^{1}(\omega) \sin n \vartheta+\right. \\
\left.+x_{n}^{2}(\omega) \cos n \vartheta\right] d \omega .
\end{gathered}
$$

Поскольку коэффициенты $x_{n}^{k}(\omega), k=1,2$, в формуле (27) зависят от параметра $\omega$ преобразования Фурье, то для вычисления эхо-сигнала 
по формуле (27) следует алгебраическую систему, состоящую из $8 \mathrm{~N}+4$ уравнений, решать на каждом шагу интегрирования. Пределы интегрирования $\omega_{A}, \omega_{B}$ в (27) как концевые точки диапазона параметра $\omega$, в котором численные значения спектральной плотности $g^{F}(\omega)$ практически отличаются от нуля, подбираются в зависимости от требуемой точности.

Выбор числа $N$ зависит от значения параметра $\omega$ и от типа функций $R(\vartheta)$. Для получения надежного результата число $N$ следует увеличивать до тех пор, пока вклады от $x_{N}^{1}(\omega)$ и $x_{N}^{2}(\omega)$ в общей сумме (27) не станут меньше требуемой точности. Следует отметить, что такая сходимость не всегда достижима $\left[{ }^{8}\right]$, поскольку при рассеянии импульса от тел некруговой формы в формировании эхо-сигнала могут участвовать наряду с расходящимися волнами и сходящиеся. Однако последние, как правило, дают заметные вклады лишь при вычислении поля вблизи рассеивателя.

\section{Л ИТ Р РА Т Р Р}

1. Bowman, J. J., Senior, T. B. A., Uslenghi P. L. E., Electromagnetic and acoustic scattering by simple shapes, Amsterdam, 1969.

2. P a o, Y. H., Mow, C. C., Diffraction of elastic waves and dynamic stress concentrations, New York, 1973.

3. И в ано в Е. А., Дифракция электромагнитных волн на двух телах, Минск, 1968.

4. К у п радз е В. Д., Основные задачи математической теории дифракции, Л.-М., 1935.

5. Коз а че к В. В., Вестн. Ленингр. ун-та, № 19, 88 (1972).

6. С в е ш н и ко в А. Г., Докл. АН СССР, Матем. Физ., 184, № 1, 63 (1969).

7. Ни кольоки й В. В., Радиотехника и электроника, № 2, 201 (1969).

8. Шенде ров Е. Л., Тр. VI Всесоюз. акуст. конф., М., 1968.

9. S h a w, R. P., J. Acoust. Soc. America, 53, No. 2, 514 (1973).

10. Кутсер М. Э., Метса в ээ Р Я. А., В сб.: Тр. Х Всесоюз. конф. по теории оболочек и пластин, 2, Тбилиси, 1975, с. 247.

Ннститут кибернетики

Академии наук Эстонской ССР
Поступила в редакцию

15/VI 1976

\section{J. METSAVEER}

\section{SUVALISE RISTLÕIKEGA SILINDRILISELT KOORIKULT SAABUVA KAJASIGNAALI ARVUTAMINE}

Esitatakse suvalise sileda ristlõikega silindriliselt koorikult saabuva tasandimpulsi kajasignaali numbrilise arvutamise algoritm, mis pōhineb Fourier' teisenduse ja BubnovGaljorkini meetodi kasutamisel. Koorikus levivaid deformatsioonilaineid kirjeldatakse Timošenko tüüpi koorikute teooria vōrranditega ja koorikut ümbritsevas vedelikus levivaid deformatsioonilaineid ideaalse kokkusurutava vedeliku võrrandiga.

\section{J. METSAVEER}

\section{ECHO-SIGNALS FROM A CYLINDRICAL SHELL WITH AN ARBITRARY CROSS-SECTION}

The algorithm of calculation of echo-signals from a cylindrical shell with an arbitrary cross-section are presented in this paper for the care of a plane incident pulse. The algorithm uses the Fourier transformation and the Bubnoff-Galyorkin method. The deformation waves in shell are described by the Timoshenko type shell theory, and those in the surrounding liquid by the acoustical approximation of the theory of ideal compressible liquid. 